\title{
Especificidad versus representatividad: enfoques metodológicos en el estudio de la migración mexicana hacia Estados Unidos
}

\author{
René M. Zenteno* \\ Douglas S. Massey**
}

Durante los últimos años hemos sido testigos de una evolución importante en los métodos de recolección de información para el análisis de los fenómenos sociales, especialmente como consecuencia de una creciente cercania entre las diversas disciplinas de las ciencias sociales. Aun con ello, existen obstáculos metodologicos no tan fáciles de hacer a un lado. Uno de ellos está relacionado con el diseño de investigaciones que puedan producir resultados con un gran nivel de especificidad sobre un problema social de carácter nacional o regional, y a la vez con un alto grado de representatividad. Por lo general, la profundidad analítica significa el sacrificio de la generalización, y viceversa. El presente trabajo constituye un análisis comparativo de dos fuentes de información con fundamentos metodológicos distintos para la medición de la migración temporal de mexicanos hacia Estados Unidos: el Proyecto sobre Migración Mexicana (Promig) y la Encuesta Nacional de la Dinámica Demográfica (Enadid). El Promig ha producido durante varios años una vasta información histórica, contextual, institucional, familiar e individual para el análisis de este fenómeno en más de 40 comunidades del país. La Enadid es una encuesta tradicional de corte transversal que recoge información sobre los factores de cambio demográfico en los niveles nacional y estatal. Mientras que la Enadid permite analizar. algunas características de la migración internacional con un gran nivel de representatividad, el Promig posibilita acercarse al entendimiento de este fenómeno con una mayor profundidad y a su análisis como proceso. Nuestro análisis comparativo señala que un diseño de investigación microsocial basado en múltiples muestras de comunidades, como el propuesto por el Promig puede contribuir a superar esta disyuntiva metodológica. Ni el análisis descriptivo de las características de la población mexicana con experiencia laboral en Estados Unidos, ni los análisis multivariados de las propensiones a viajar a Estados Unidos por motivos laborales, arrojan evidencias sobre la existencia de sesgos significativos en el Promig al comparar sus estadísticas con las de la Enadid. Si bien no es posible señalar que por ello los datos del Promig son "representativos" de la región más tradicional de la migración México-Estados Unidos, sí es posible concluir sobre los alcances de generalización de los estudios basados en los mismos. El trabajo empírico demuestra además los problemas de selectividad y especificidad en que encuestas tradicionales como la Enadid

* Centro de Estudios Estratégicos, Instituto Tecnológico y de Estudios Superiores de Monterrey, Campus Guadalajara.

** Profesor de la Universidad de Pennsylvania. 
incurren por seleccionar sólo migrantes internacionales residentes en México, y por intentar capturar un proceso socioeconómico tan complejo con un número limitado de variables. ${ }^{1}$

El problema de investigación

La migración internacional es un fenómeno social, económico y demográfico de gran relevancia para México y Estados Unidos. Entre 1980 y 1990, 37\% del crecimiento neto de la población de Estados Unidos fue producto de este componente demográfico (Passel y Edmonston, 1994), y durante los próximos años dos terceras partes de dicho crecimiento tendrá como origen el arribo de nuevos migrantes y su fecundidad (Fix y Passel, 1994). En este contexto México se ha constituido en la fuente más importante de inmigración en Estados Unidos. Durante los años sesenta la migración mexicana documentada totalizó 430 mil personas. En la década siguiente esta cifra aumentó a 680 mil, y para los años ochenta había alcanzado un número más que notorio de 3 millones (U.S. Immigration and Naturalization Service, 1992). Woodrow y Passel (1990) calculan que otros 800 mil mexicanos arribaron sin documentos entre 1980 y 1990 , y estadísticas oficiales muestran que alrededor de 12 millones entraron a Estados Unidos como visitantes temporales (U.S. Immigration and Naturalization Service, 1992). Más aún, durante la primera mitad del presente decenio un número adicional de 2.2 millones de mexicanos legales arribaron a Estados Unidos, excediendo con ello el ritmo récord establecido en la década anterior (U.S. Immigration and Naturalization Service, 1996).

Los números anteriores dejan entrever que la migración internacional no es un fenómeno demográfico y socioeconómico soslayable para México. En términos demográficos, la emigración desde el país es ya un factor significativo en la reducción de su crecimiento demográfico (Corona, 1993). Según el Programa Nacional de Población (PEFEUM, 1995), México pierde alrededor de 290 mil personas al año debido a la emigración, reduciendo con ello en $16 \%$ su tasa de crecimiento. En términos económicos, los "migradólares" enviados por los mexicanos que trabajan en Estados Unidos alcanzaron la cifra de

\footnotetext{
${ }^{1}$ Las personas interesadas en reproducir el análisis empírico de este trabajo, pueden solicitar las bases de datos necesarias para ello a cualquiera de los autores.
} 
2 mil millones de dólares a finales de los años ochenta (Keely y Tran, 1989; Lozano, 1993; Massey y Parrado, 1994), y rebasaron los 4 mil millones a la mitad de la década siguiente (Lozano y McClellan, 1997). ${ }^{2}$

A pesar de la obvia importancia de la migración internacional para ambos países, la discusión de este tema es una labor complicada en el contexto de las relaciones México-Estados Unidos. Fundamental para ello no es sólo el hecho de que México es la principal fuente de origen de la migración hacia Estados Unidos, sino también de que la emigración desde este país es predominantemente clandestina. Tres cuartas partes de los 3.2 millones de migrantes -previamente indocumentados- que obtuvieron su residencia con la ley de inmigración de 1986 en Estados Unidos eran mexicanos (U. S. Immigration and Naturalization Service, 1990). A pesar de esta legalización masiva, cerca de un millón de mexicanos continuaban residiendo en Estados Unidos en 1992 sin permiso legal para hacerlo, constituyéndose así en $31 \%$ de la población indocumentada (Fix y Passel, 1994). ${ }^{3}$

Como en el estudio de cualquier fenómeno social complejo, el acceso a información confiable y de calidad es crucial para el entendimiento de las causas y consecuencias del fenómeno migratorio México-Estados Unidos. La naturaleza temporal e indocumentada de la gran mayoría de estos movimientos impone serias restricciones a los esfuerzos de recolección de información. Además, las políticas recientes implantadas por el Congreso estadunidense para una mayor persecución y detención de los migrantes clandestinos han conducido a éstos a ocultarse aún más, haciendo con ello más difícil la medición y el estudio de este fenómeno. En general, el carácter transitorio, circular e indocumentado de la migración México-Estados Unidos limita seriamente el uso de enfoques metodológicos tradicionales de recolección de datos para su estudio, y disminuye significativamente el aprovechamiento de fuentes convencionales de información como son los censos y los registros administrativos.

El censo de población de Estados Unidos capta un número significativo de personas nacidas en México, sin embargo, éste no ofrece

\footnotetext{
${ }^{2} \mathrm{Si}$ se toman en cuenta sus efectos multiplicativos, el gasto corriente y la inversión producto de estas remesas generan adicionalmente alrededor de 12 mil millones de dólares en ingresos y 13 mil millones de dólares en producción (Durand, Parrado y Massey, 1995).

${ }^{3}$ Warren (1995) estima la población mexicana indocumentada en poco más de 1.4 millones de personas, cifra que representa $40 \%$ del total. Según estimaciones más recientes de Passel (1995), alrededor de 150 mil mexicanos continuaron arribando sin documentos legales a Estados Unidos a principios de la década actual.
} 
ninguna información sobre su carácter legal de residencia y subestima tanto a la población documentada como indocumentada (especialmente a esta última). Aunque es posible construir cohortes sintéticas de inmigrantes según periodos de llegada, la información censal es de tipo transversal, y sufre de muchas limitaciones metodológicas como resultado de una emigración selectiva y de la calidad cambiante de las cohortes (Borjas, 1985, 1987, 1991; Lindstrom y Massey, 1994). Adicionalmente, el censo proporciona muy pocos datos sobre las características de los inmigrantes previamente a su asentamiento en Estados Unidos, y su naturaleza decenal no garantiza contar en todo momento con información actualizada. ${ }^{4}$

El Servicio de Inmigración y Naturalización (SIN) constituye una fuente potencial de información para el estudio de la inmigración mexicana. Esta agencia federal estadunidense produce reportes anuales con información proveniente de las solicitudes de residencia permanente en dicho país. La información disponible incluye edad, sexo, país de nacimiento, país de última residencia, ocupación, lugar en que se intenta residir, y tipo de admisión de los solicitantes. Desafortunadamente no se averigua sobre factores tan importantes como la educación (Jasso y Rosenzweig, 1990), y existe muy poca información sobre la situación de los migrantes una vez que entran a Estados Unidos, y sobre el número y las características de aquellos que dejaron este país (Jasso y Rosenzweig, 1982; Warren y Kraly, 1985; Woodrow, 1988)..$^{5}$

El SIN publica también datos reportados por la patrulla fronteriza sobre los extranjeros detenidos y sujetos a deportación. La información agregada de estas detenciones ha sido utilizada para me-

\footnotetext{
${ }^{4}$ Este último problema de temporalidad es parcialmente superado por la Encuesta Continua de Población de Estados Unidos (Current Population Survey), la cual además incluye ahora preguntas sobre el lugar de nacimiento y la fecha de entrada a este país de los inmigrantes. No obstante ello, esta fuente de información adolece de los mismos problemas metodológicos del censo de población, y sufre el problema adicional de contar con muestras muy pequeñas de inmigrantes.

5 Otro problema serio es que el SIN únicamente recolecta información sobre migrantes mexicanos cuando éstos se convierten en residentes legales, y no cuando entraron al país. En 1994, por ejemplo, 39\% de todos los "inmigrantes" ya estaban en Estados Unidos (como estudiantes, visitantes, trabajadores temporales, empleados, asilados o refugiados). Adicionalmente, un número desconocido pero presumiblemente grande de migrantes residían en Estados Unidos sin documentos legales para ello. Por ejemplo, el estudio de Portes (1979) mostró que del total de migrantes mexicanos legales recién llegados a Estados Unidos en 1972-1973, 62\% había vivido en este país de forma indocumentada.
} 
dir la migración neta indocumentada. Sin embargo, sus estadísticas presentan limitaciones de importancia. Primero, porque éstas son extremadamente sensibles a cambios en los esfuerzos de aprehensión de la patrulla fronteriza, y al número de intentos de entrada clandestina por parte de la población migrante (véase Bustamante, 1989; Bean et al., 1990; White, Bean y Espenshade, 1990; Espenshade, 1990 y 1994; Corona, 1993; Espenshade y Acevedo, 1995). Segundo, porque proporcionan muy pocos datos sobre las características y el comportamiento de la población indocumentada (Davidson, 1981; Hill, 1985). ${ }^{6}$

Las fuentes convencionales mexicanas también presentan problemas. Los censos de población de México históricamente han medido como migraciones cambios de residencia habitual, por lo que los movimientos temporales a Estados Unidos muy pocas veces son reportados. Aun en el caso de que se deseara analizar las migraciones de carácter más permanente a Estados Unidos, los censos más recientes han mostrado muy poca continuidad en la definición conceptual y temporal de éstas (Corona, 1990 y 1991). Sin embargo, lo que parece más sorprendente es que la fuente de información con mayor cobertura nacional todavía no haya incluido preguntas específicas que permitan cuantificar, directa o indirectamente, la migración temporal y definitiva a Estados Unidos.

Frente a estas limitaciones, los científicos sociales interesados en el estudio de la migración México-Estados Unidos han intentado medios alternativos para la recolección de datos. La naturaleza indocumentada y temporal de la mayoría de estos movimientos prepondera los enfoques metodológicos desde el lado de la oferta de mano de obra sobre aquéllos desde el lado de la demanda. La producción de información en México hace menos complicada la medición y caracterización de la migración temporal indocumentada a Estados Unidos, además de que permite el estudio adecuado de las causas y consecuencias de este fenómeno en los lugares de origen a la luz de los contextos regionales, comunitarios y familiares en que tiene lugar.

\footnotetext{
6 Otras fuentes de información importantes en Estados Unidos son varias enuestas nacionales que recogen información longitudinal (National Longitudinal jurveys, High School and Beyond Survey, entre otras). A pesar de su importancia, us muestras no cuentan con un número suficiente de inmigrantes que permita aborlar adecuadamente el estudio de este fenómeno.
} 
Actualmente, el Proyecto sobre Migración Mexicana (Promig) y la Encuesta sobre Migración en la Frontera Norte de México (Emif) se han constituido como las fuentes de información más importantes para el estudio de la migración México-Estados Unidos desde la perspectiva de la oferta de mano de obra mexicana. Ambas parten de objetivos y bases metodológicas muy distintos. La Emif tiene como propósito la medición y caracterización directa de los flujos migratorios laborales internacionales entre México y Estados Unidos, y entre las localidades fronterizas y el resto del país (Colef, Conapo y STPS, 1994). Partiendo de una metodología de poblaciones móviles, la Emif representa la fuente de información más relevante para aproximarse a la medición anual de los desplazamientos entre México y Estados Unidos (y por lo tanto del saldo neto que resulta de la diferencia entre los mismos), a la caracterización demográfica y socieconómica de la población en movimiento, a los determinantes y tendencias del flujo, y a algunos impactos de las medidas de la política migratoria del gobierno estadunidense.

No obstante que el diseño muestral de la Emif representa un avance importante para la cuantificación de la migración MéxicoEstados Unidos, la operacionalización del mismo impone serias restricciones al estudio de este fenómeno como un proceso social y económico. Debido a que las entrevistas se realizan con pasajeros en tránsito en aeropuertos, centrales de autobuses, etc., el cuestionario necesariamente tiene que limitarse a averiguar sobre muy pocos factores asociados con los determinantes de esta movilidad geográfica. Además, es importante tomar en cuenta que la emigración a Estados Unidos se define a partir de una declaración de intención del entrevistado a hacerlo, y no del hecho mismo de haberlo logrado. ${ }^{7}$

El Proyecto sobre Migración Mexicana (Promig), también conocido por sus iniciales en inglés MMP (Mexican Migration Project), es un esfuerzo binacional conducido por la Universidad de Guadalajara y la Universidad de Pennsylvania. Este proyecto de investigación tiene sus orígenes conceptuales y metodológicos en un estudio realiza-

\footnotetext{
${ }^{7}$ En nuestra opinión, la dinámica de entrevista muy probablemente explique el bajo porcentaje de mujeres captado por la Emif en el flujo hacia Estados Unidos (menos de 5\%). Por un lado, las mujeres son más reacias que los hombres a aceptar una entrevista en el momento preciso de haber arribado a la frontera norte (prácticamente descendiendo de un avión o camión). Por el otro, es muy factible que las mujeres tengan más temor que los hombres a declarar en un espacio público sus intenciones de cruzar a Estados Unidos.
} 
do en cuatro comunidades de la región centro-occidente de México a principios de los años ochenta, cuyos principales resultados fueron publicados en el libro Return to Aztlán (Massey et al., 1987).

A diferencia de otros proyectos que tienen como único interés la cuantificación y caracterización del filujo o la población migrante hacia Estados Unidos, el Promig constituye un esfuerzo demográfico, sociológico y antropológico para el entendimiento de esta migración como un proceso social y económico. La información pública del Promig permite: a) comparar las características y el comportamiento de migrantes documentados e indocumentados; $b$ ) medir variaciones temporales en las características de ambos grupos; $c$ ) estudiar el proceso de la migración a partir de las historias de vida de las personas; $d$ ) analizar los antecedentes y las características de los migrantes antes y después de cruzar a Estados Unidos; $e$ ) estudiar las transiciones entre diferentes estadios de condición legal para ingresar a Estados Unidos, y modelar diferentes tipos de movimientos hacia y desde dicho país, y $f$ ) proveer una fuente continua de información longitudinal capaz de monitorear los efectos de las políticas mexicanas y estadunidenses.

El proyecto ha realizado encuestas en hogares de varias comunidades en México y lugares de destino de los migrantes en Estados Unidos durante los últimos diez años. Actualmente la base de datos del Promig cuenta con muestras disponibles de 39 comunidades (la mayoría pertenecientes a los estados con mayor tradición migratoria hacia Estados Unidos), y de 5063 migrantes internacionales (58\% de los cuales ingresaron en Estados Unidos sin documentos legales en su viaje más reciente).

La información proporcionada por el Promig es probablemente a más vasta para el estudio de la migración mexicana hacia Estados Unidos. Sin embargo, los datos de este proyecto han sido criticados jor sus problemas de generalización (Bustamante, 1998). Por un lado, jorque las entidades federativas incluidas en la selección de comunidades del proyecto cubren principalmente la región centro-occiden:e, por lo que sus resultados no pueden extenderse a todo el país. Por 21 otro, porque aunque los hogares encuestados son seleccionados orobabilísticamente en el interior de cada comunidad, las comunidades en sí mismas no son seleccionadas aleatoriamente al interior de a región. En consecuencia, los datos no son en sentido estricto "rerresentativos" de la población de la región. El Promig intenta supear la primera limitación con la inclusión de estudios de casos en 
otras regiones del país. ${ }^{8}$ Sin embargo, la segunda limitación es más sustantiva pues es intrínseca a la metodología del proyecto.

¿En qué medida la búsqueda de una mayor especificidad del proceso de la migración México-Estados Unidos en el Promig ha significado un sacrificio revelador de sus alcances de generalización? ¿En qué sesgos incurre el Promig por adoptar un diseño de investigación basado en el estudio de comunidades en vez de uno basado en grandes agregados poblacionales? Las anteriores preguntas constituyeron el principal fundamento del presente trabajo de investigación. Como es de suponerse, la evaluación empírica de estas preguntas requería de la disponibilidad de una fuente de información alternativa que cumpliera necesariamente con dos características: contar con una muestra probabilística de hogares con representatividad nacional y regional, e identificar directamente residentes mexicanos con experiencia migratoria en Estados Unidos. La reciente accesibilidad a los datos de la Encuesta Nacional de la Dinámica Demográfica (Enadid), realizada en 1992, representó una excelente oportunidad para realizar esta labor. ${ }^{9}$ La Enadid fue la primera encuesta demográfica nacional capaz de producir estimaciones confiables en el nivel estatal. Aunque no fue diseñada específicamente para estudiar la migración internacional, su cuestionario incluyó varias preguntas que permiten la identificación de trabajadores internacionales de manera comparable con el Promig.

Así pues, el objetivo general de nuestra investigación fue evaluar las principales fuentes de error originadas por las particularidades metodológicas del Promig, sobre todo en lo que respecta a la medición de las características de la población mexicana con experiencia laboral en Estados Unidos. En nuestra opinión, esta labor analítica no es únicamente relevante para el estudio mismo del fenómeno migratorio México-Estados Unidos, sino también para la apreciación de diseños de investigación alternativos para el análisis de fenómenos sociales. La comparación de la información del Promig y la Enadid permite examinar, en el contexto específico de la migración internacional, los alcances de investigaciones cuyo fundamento metodológico

${ }^{8}$ El Promig contará en los próximos meses con información de más de 54 comunidades, entre las que se incluyen muestras de los estados de Aguascalientes, Baja California, Colima, Guerrero, Morelos, Oaxaca, Puebla y Sinaloa.

${ }^{9} \mathrm{La}$ comparación de los datos del Promig con los datos de la Emifi resulta insustancial dadas sus grandes diferencias metodológicas. 
es el estudio sistemático de comunidades, a la luz de resultados que tienen como base muestras de grandes agregados poblacionales.

Haciendo uso de comparaciones estadísticas directas, tanto univariadas como multivariadas, nuestro análisis permite concluir que si bien existen discrepancias entre el Promig y la Enadid, éstas son mínimas considerando sus diferencias metodológicas. Por un lado, el Promig reporta una mayor prevalencia de migración temporal a Estados Unidos que la observada con los datos de Enadid. Sin embargo, estas diferencias son resultado de las distintas formas en que estas encuestas identifican a los migrantes internacionales, más que de variaciones reales en la prevalencia de la migración. Por otro lado, y a pesar de que el Promig reporta mayores proporciones de migrantes solteros, con mayor nivel educativo y que no son jefes de hogar, ambas fuentes de información reportan una gran similitud en la composición por edad y sexo de los trabajadores internacionales, así como en la duración de los viajes a Estados Unidos. La sorprendente correspondencia entre la Enadid y el Promig es confirmada por los resultados multivariados prediciendo la propensión a migrar hacia Estados Unidos por motivos laborales.

No obstante los propósitos propiamente metodológicos del análisis, los resultados presentados permiten generar tres conclusiones. Primera, el proceso de la migración a Estados Unidos parece presentar homogeneidad en términos de sus características independientemente del tamaño de localidad de residencia de los migrantes, por lo menos en el interior de la región centro-occidente del país. Esta conclusión deriva del hecho de que las discrepancias entre el Promig y la Enadid, en términos de población muestreada según tamaño de localidad de residencia, no producen diferencias significativas en la caracterización de la migración laboral mexicana hacia Estados Unidos; esto tanto en un contexto de análisis descriptivo como multivariado. Segunda, los modelos estadísticos multivariados muestran claramente la importancia y necesidad de corregir dos problemas fundamentales en el estudio de la migración México-Estados Unidos: el sesgo de selectividad producto de incluir sólo migrantes que viven regularmente en México, y el error de especificación resultado de intentar capturar in proceso socioeconómico tan complejo con un número limitado de variables. Finalmente, este trabajo de investigación muestra que estudios basados en el análisis sistemático de comunidades pueden ser zxitosos en la extensión de sus generalizaciones para una región deerminada, además de ofrecer una mayor especificidad para el ententimiento de muchos procesos sociales. 
Dos enfoques metodológicos para la medición de la migración internacional

Proyecto sobre Migración Mexicana (Promig)

La metodología subyacente al Promig ha sido la "etnoencuesta", la cual simultáneamente pone en práctica métodos de investigación etnográfica y de encuesta en comunidades mexicanas estratégicamente seleccionadas (Massey et al., 1987 y Massey, 1987). La visión del proyecto es que las metodologías cualitativa y cuantitativa son complementarias más bien que excluyentes, y que debidamente combinadas las debilidades de una se convierten en la fortaleza de la otra. Así, es posible producir información de mayor confiabilidad y consistencia interna que si se trabajara únicamente con una de ellas (Massey, 1994). Este principio básico se refileja en todas las etapas de recolección de información del Promig: diseño del cuestionario, diseño muestral, procedimientos de entrevista y organización de las bases de datos.

La base de datos del Promig incluye actualmente cinco comunidades encuestadas en el estudio original de 1982, y 34 comunidades muestreadas entre 1987 y 1995 con procedimientos comparables. Los datos provienen de muestras representativas obtenidas durante los meses de invierno en comunidades localizadas en su mayoría en los estados de Jalisco, Michoacán, Guanajuato, Nayarit y Zacatecas, los que de hecho conforman la región de donde tradicionalmente ha salido el mayor número de migrantes internacionales (Conapo, 1986; Jones; 1988). Las comunidades han sido seleccionadas para representar situaciones muy variadas en lo que respecta a su tamaño, geografía, etnicidad y organización económica. Así, el proyecto incluye microestudios de pequeñas villas y pueblos agrícolas, localidades industriales pequeñas y medianas, comunidades dedicadas a la minería o a la pesca, centros turísticos, centros comerciales regionales y una variedad de comunidades en el caso de las áreas metropolitanas.

El cuadro 1 presenta información sobre estas comunidades, y el mapa 1 muestra las entidades federativas en que el Promig ha centrado su trabajo y la ubicación geográfica de las localidades, respectivamente. Las comunidades varían en un rango poblacional de 300 a 3 millones de personas, produciendo un tamaño promedio de $92 \mathrm{mil}$ personas. Los hogares son seleccionados utilizando un procedimiento de muestreo aleatorio simple en el interior de cada comunidad, 
utilizando para ello marcos muestrales construidos a partir de un censo de viviendas de la comunidad. El escrutinio es por lo regular exhaustivo en ranchos, pueblos y ciudades pequeñas. Sin embargo esta labor es imposible en las grandes áreas urbanas, por lo que en estas localidades se han estimado muestras a partir de la preselección de una colonia o barrio (estas muestras son indicadas con un asterisco en el cuadro 1). Los tamaños de muestra se ubican entre 100 y 400 hogares por cada comunidad. Las fracciones muestrales (005 a .999) dependen principalmente del tamaño de la comunidad. La más pequeña de ellas representa una muestra aleatoria de una zona metropolitana, mientras que la más grande corresponde prácticamente a un censo de una comunidad rural pequeña. Hasta el momento, 7293 hogares han sido encuestados e incluidos en la base de datos.

Las entrevistas generalmente se llevan a cabo durante los meses de diciembre y enero, ya que en estos meses es más probable entrevistar a migrantes temporales en México pues muchos de ellos regresan a pasar las fiestas de Navidad con sus familias. ${ }^{10}$ La información recolectada en México se complementa con muestras no representativas de migrantes establecidos en Estados Unidos, realizadas en el verano subsiguiente al invierno en el que se levantó la encuesta. A partir del trabajo de campo en México se determinan los puntos de destino de los migrantes en Estados Unidos, y se envían encuestadores a estas áreas para entrevistar a los migrantes establecidos. Con ello, las entrevistas en Estados Unidos implican un método de muestreo de "bola de nieve" (Goodman, 1961). En la mayoría de las comunidades de la diáspora se realizaron 20 entrevistas, salvo algunas excepciones en donde se realizó un número menor. ${ }^{11}$ Una vez agrupadas y contrapesadas, las muestras en ambos países ofrecen un retrato comprensivo de las comunidades binacionales, creadas éstas mediante el proceso recurrente de la migración internacional y del establecimiento en Estados Unidos.

\footnotetext{
${ }^{10}$ En algunos casos este periodo de referencia varía cuando el trabajo de campo preliminar revela que los migrantes regresan durante otro periodo del año (comúnmente durante el verano o durante las fiestas religiosas del pueblo).

${ }_{11}$ Aunque estas muestras no son estrictamente representativas de las comunidades en la diáspora, se ha desarrollado un conjunto de ponderadores para inferir su contribución relativa al total de la muestra binacional. Los contrapesos vienen a ser el inverso de la fracción muestral de cada lugar (Massey y Parrado, 1994).
} 


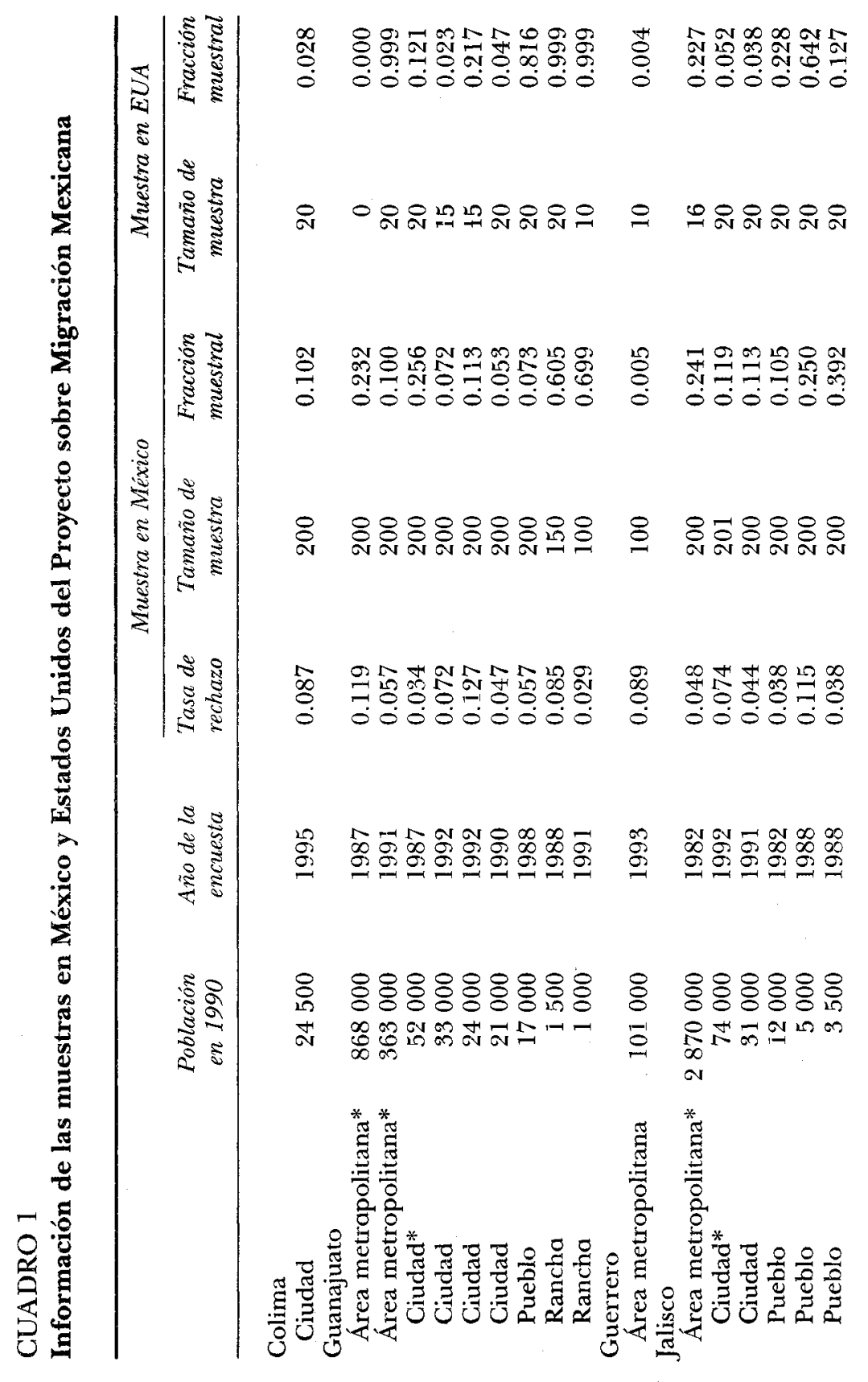




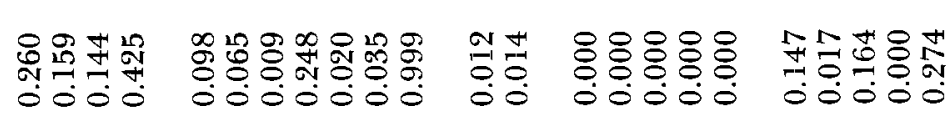

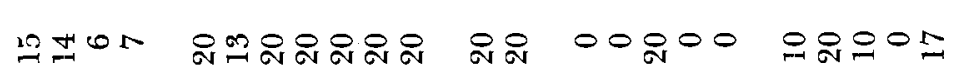

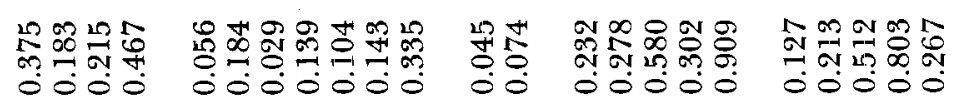

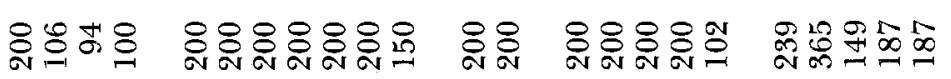

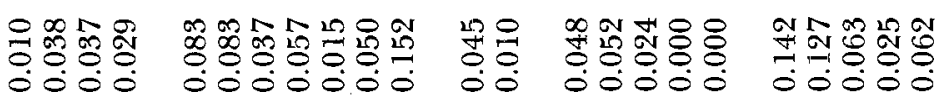

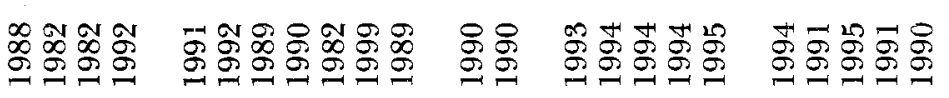

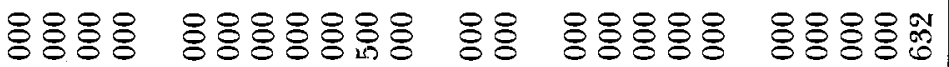
m mov

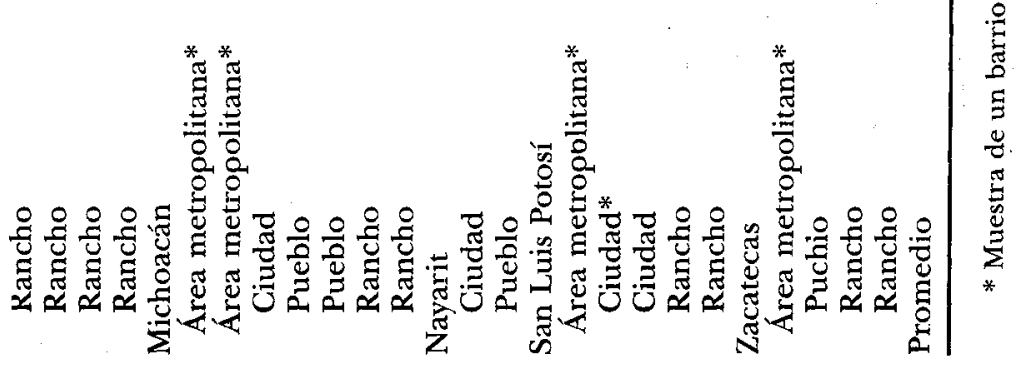







Como se mencionó, los informantes del Promig son entrevistados utilizando los métodos propios de la etnoencuesta. De todos los miembros del hogar se recolecta información sobre sus características sociales y demográficas generales, sobre su historia migratoria y, de proceder, sobre su primer y último viaje a Estados Unidos. De cada jefe de familia se obtiene además información detallada sobre su historia de vida, que incluye una historia laboral, una historia migratoria, una historia de negocios y propiedades, una historia marital y una historia de fecundidad. Por otra parte, se realiza un inventario de información básica sobre cada localidad, en donde se retoma información sobre aspectos sociales, económicos y demográficos en diferentes periodos de tiempo. De igual modo se hace un recuento de los cambios en la infraestructura de cada localidad a partir de información recabada en archivos y entrevistas con informantes y autoridades locales. Por último, los datos macroeconómicos se compilan de datos publicados por el Fondo Monetario Internacional de 1994. Las historias de vida se combinan con las historias locales y los datos estadísticos macroeconómicos para crear los archivos básicos en el nivel de la comunidad, del hogar e individual.

\section{Encuesta Nacional de la Dinámica Demográfica (Enadid)}

La Encuestra Nacional de la Dinámica Demográfica, a diferencia del Promig, es una encuesta completamente estructurada con grandes alcances de representación muestral, pero que no fue diseñada conceptualmente para estudiar con profundidad una problemática demográfica específica. La Enadid fue realizada por el Instituto Nacional de Estadística, Geografía e Informática (INEGI) con el objetivo de producir datos sociodemográficos representativos no sólo en el nivel nacional, sino también de cada una de las entidades federativas del país y de sus áreas rurales y urbanas. La Enadid contó con una muestra probabilística, representativa, multietápica y estratificada de 64 mil hogares distribuidos de manera uniforme en cada uno de los estados y el Distrito Federal. ${ }^{12} \mathrm{El}$ levantamiento de la información tuvo lugar durante los meses de septiembre, octubre y noviembre de 1992. El tamaño de muestra final fue de 57916 hogares que produje-

12 La estratificación en el diseño muestral de la Enadid implica el uso de factores de expansión con el fin de producir estimaciones no sesgadas de las variables. 
ron información sobre 277552 personas, para una tasa de respuesta de un poco más de $90 \%$ (INEGI, 1994).

La Enadid teóricamente capta a todas las personas que residen "normalmente" en los hogares, aun cuando éstos estén trabajando o estudiando temporalmente en algún otro lugar. Por lo tanto, un migrante temporal en Estados Unidos es reportado e incluido en la muestra siempre y cuando aún exista un hogar en México que lo pueda listar como residente. Con respecto a la migración, la encuesta averigua el lugar de nacimiento de los miembros del hogar, su condición de residencia en otro lugar aun cuando haya sido por un corto tiempo, su tiempo de residencia en el lugar actual, su lugar de residencia anterior si migró alguna vez durante su vida, y su lugar de residencia en 1987 (sólo miembros de cinco años y más). Además, para los miembros mayores de 11 años de edad, se preguntó sobre la condición de trabajo o búsqueda de trabajo en Estados Unidos alguna vez durante sus vidas, y en caso afirmativo las fechas de salida y retorno de su último viaje a dicho país.

La Enadid ofrece varias posibilidades para identificar migrantes mexicanos con experiencia en Estados Unidos: 1) personas que nacieron en México cuyo último lugar de residencia fue Estados Unidos; 2) personas de cinco años y más de edad que nacieron en México y vivían en Estados Unidos en 1987; 3) personas miembros del hogar (vivan "normalmente" o no en el hogar) que fueron a Estados Unidos entre 1987 y 1992, y 4) personas de 12 años y más que han trabajado o buscado trabajo en Estados Unidos.

Las primeras dos opciones tienen serias limitaciones pues se refieren a una población migrante que consideró Estados Unidos como un lugar de residencia habitual, subestimando de manera importante a los migrantes temporales o circulares. Además no es posible distinguir a los migrantes laborales de aquellos que migraron por otras razones (estudios, negocios, etc.). El tercer método captura tanto migrantes permanentes como temporales en la medida que existía un miembro del hogar en México que los declara como tal a finales de 1992. Sin embargo, la restricción al periodo 1987-1992 disminuye sustantivamente el número de migrantes potenciales, y limita su utilidad como base de comparación con el Promig.

Por lo tanto, la última opción se consideró como la más adecuada para este ajercicio metodológico de evaluación. Primero, porque la mitad del total de migrantes reportados con el método anterior son también captados como migrantes laborales. Segundo, porque 
permite trabajar con una definición de migrantes laborales (la principal fuente de migración a Estados Unidos) clara y comparable entre ambas fuentes. Tercero, porque permite obtener un tamaño de muestra suficientemente grande para el análisis, produciendo con ello pruebas estadísticas más precisas y confiables. Cuarto, porque la información sobre el calendario del último viaje a Estados Unidos permite examinar la duración de los mismos.

\section{Bases de comparación y consideraciones metodológicas}

La comparación entre el Promig y la Enadid se fundamenta en diversos factores. Ambas fuentes de información cuentan con una base metodológica y muestral de hogares. Desde la perspectiva de la oferta de mano de obra, esta similitud permite un entendimiento adecuado de la migración temporal documentada e indocumentada de mexicanos hacia Estados Unidos. Ambas encuestas producen información comparable sobre un número importante de características sobre la migración desde la región centro-occidente, la más importante en el origen de la migración mexicana al país del norte.

Sin embargo, como se ha podido constatar existen diferencias metodológicas de consideración entre la Enadid y el Promig que, teóricamente, deberían producir resultados distintos en el estudio de la migración laboral México-Estados Unidos. Por un lado, la Enadid adoptó un diseño muestral cuyo propósito fue garantizar la representatividad de las variables de hogar e individual en los niveles nacional y estatal, mientras que el Promig garantiza esta representación en el nivel de cada una de las comunidades y por derivación de su conjunto. Con propósitos únicamente ilustrativos, podría argumentarse que la Enadid adopta un procedimiento de muestreo de natura leza deductiva, mientras que el Promig adopta uno de carácter más inductivo. La idea detrás del Promig ha sido la de construir una amplia base de información añadiendo microestudios de diversas comunidades para la región más tradicional de la migración MéxicoEstados Unidos en un primer momento, y para otras regiones del país en la actualidad. Encuestas como la Enadid son capaces de producir información precisa de la estructura sociodemográfica para grandes agregados de población, pero proporcionan muy poca información que permita estudiar con profundidad los procesos sociales y económicos que producen dicha estructura. 
Por otro lado, los periodos de referencia del Promig y la Enadid son también distintos. La información del Promig proviene de muestras probabilísticas de comunidades recabadas durante 1982-1983 y 1987-1994, mientras que la Enadid fue levantada durante los últimos meses de 1992. El largo periodo de referencia del Promig hace a la información de este proyecto más sensible a rápidos cambios estructurales como han sido las crisis económicas del país y los cambios en las políticas de inmigración de Estados Unidos (i.e. IRCA). El registro de historias de vida en el Promig compensa en gran medida esta limitación, pues es posible comparar las diferentes muestras según la referencia temporal de los fenómenos que se quieran analizar.

$\mathrm{El}$ análisis de este trabajo se centra en la migración laboral y temporal a Estados Unidos. Las razones para ello son varias. Primera, porque esta migración constituye todavía la más importante fuente de movimientos de personas entre México y Estados Unidos y, como se señaló anteriormente, las características metodológicas de la Enadid y el Promig permiten analizar este tipo de migración de forma adecuada. Segunda, porque ninguna de estas fuentes de información restringió la condición de migración laboral a Estados Unidos a un periodo explícito. Finalmente, porque ambas encuestas proporcionan información sobre el calendario del último viaje a Estados Unidos, por lo tanto es posible ubicar temporalmente estas migraciones y determinar su duración. En consecuencia, en el presente estudio, migrantes temporales hacia Estados Unidos se refiere a todos los residentes en México de 12 años de edad y más que han estado en Estados Unidos, ya sea trabajando o buscando trabajo, una o más veces durante sus vida.

La Enadid capta directamente como migrantes laborales internacionales a los residentes habituales en México que han ido a Estados Unidos por motivos laborales. Para adoptar una definición comparable con el Promig fue necesario excluir a todos los migrantes mexicanos incluidos en las muestras de Estados Unidos, así como aquellos mexicanos en las muestras de México que no eran miembros regulares de los hogares. También fue necesario excluir a los niños y adultos que habían migrado a Estados Unidos por otros motivos que no fueran laborales (familiares, estudios, etcétera). ${ }^{13}$

${ }^{13}$ Las muestras en Estados Unidos son tomadas en cuenta sólo en la parte final del trabajo. 
Así pues, la unidad de análisis en este trabajo son los migrantes temporales mexicanos con experiencia laboral en Estados Unidos. El método analítico y de presentación que se utiliza para el desarrollo de los objetivos de este trabajo es el siguiente. Primero se hace uso de la Enadid para examinar los sesgos incurridos por el Promig en lo que respecta a la cobertura geográfica y a la medición de la prevalencia de la migración laboral México-Estados Unidos, y se contrastan estadísticas univariadas del perfil sociodemográfico de la población migrante reportada por ambas fuentes de información. Los resultados que se analizan en esta primera etapa hacen referencia tanto a las muestras totales de la Enadid y el Promig, como a las muestras de un grupo seleccionado de entidades federativas de la región centro-occidente del país para las cuales el Promig cuenta con un número significativo de comunidades estudiadas.

En una segunda etapa del trabajo, el ejercicio de evaluación metodológica adquiere mayor rigurosidad analítica al estimar modelos multivariados de regresión para estudiar los determinantes individuales y familiares de migrar a Estados Unidos por motivos laborales. Por un lado, se seleccionó y evaluó un modelo básico tomando en consideración el número limitado de variables disponibles en la Enadid para estudiar este fenómeno. Este mismo modelo fue calculado con la información del Promig con el fin de discutir en qué medida sus resultados presentan problemas de generalización en comparación con los estimados con los datos representativos de la Enadid. Por otro lado, los datos del Promig se utilizan para ejemplificar los problemas de selectividad y especificidad en que incurren encuestas como la Enadid. Para ello se calcularon dos modelos más de regresión logística con la información del Promig. En el primero la ecuación básica se estima nuevamente pero tomando además en consideración las características de los migrantes que se han asentado de forma más permanente en Estados Unidos; en el segundo, esta ecuación se expande para incluir otras características sociales asociadas teóricamente con la migración internacional.

Distribución geográfica y prevalencia de la migración

En 1992, según las estimaciones de la Enadid, cerca de 2.5 millones de residentes mexicanos habían estado en Estados Unidos ya sea trabajando o buscando trabajo (8 765 casos en la muestra sin ponde- 
rar), los cuales representaban $4.1 \%$ de la población nacional de 12 años y más (véase el cuadro 2) ${ }^{14}$ Desde principios de este siglo, unos cuantos estados de México han sido responsables de la mayoría de la migración hacia Estados Unidos (véase Gamio, 1930; Dagodag, 1975; North y Houston, 1976 y Jones, 1988). La alta prevalencia de la migración internacional en la región centro-occidente del país es más que evidente: la concentración de la población total de trabajadores internacionales en esta región es dos veces superior a la expresada por la distribución geográfica de la población nacional. Aproximadamente $43 \%$ de los migrantes laborales captados por la Enadid eran residentes habituales de las entidades federativas de Aguascalientes, Colima, Guanajuato, Jalisco, Michoacán, Nayarit, San Luis Potosí y Zacatecas, mientras que en este conjunto de estados sólo residía $22 \%$ de la población de 12 años y más del país. ${ }^{15}$

Los datos del cuadro 2 muestran claramente que el diseño muestral del Promig produce una distribución regional sesgada de la migración laboral México-Estados Unidos, al comparar los datos de esta encuesta con una muestra representativa nacional como la Enadid. Los datos del Promig están sumamente concentrados en la región centro-occidente. De los 37817 mexicanos de 12 años y más que reportaron en esta encuesta haber estado en Estados Unidos por motivos laborales en algún momento de sus vidas (4 255 casos en la muestra sin ponderar), $83 \%$ residía en las entidades federativas previamente mencionadas. Este resultado es lógicamente producto del hecho de que 38 de las 39 comunidades del Promig se localizan en esta zona del país. ${ }^{16}$

14 Aunque la información no se proporciona en ningún cuadro, es importante mencionar que 6\% de estos migrantes estaban en Estados Unidos en el momento de la encuesta, y $3 \%$ eran residentes de la frontera norte de México que cruzaban cotidianamente a trabajar a Estados Unidos.

15 En un trabajo reciente probamos empíricamente que, por lo menos hasta 1993, la importancia relativa de la región en el proceso de la migración temporal México-Estados Unidos había presentado muy pocas variaciones temporales (Durand, Massey y Zenteno, 1998).

${ }^{16} \mathrm{Al}$ momento de escribir este documento, la experiencia migratoria del resto del país está representada por la muestra de un área metropolitana del estado de Guerrero. Sin embargo, como se comenta en una nota de pie de página anterior, varias comunidades de otros estados han sido o serán agregadas a la base de datos del Promig. 


\section{CUADRO 2}

Distribución de la población total, de la población de 12 años y de más edad y de los trabajadores mexicanos con experiencia laboral en Estados Unidos por entidades federativas de la región centro-occidente, 1992

\begin{tabular}{|c|c|c|c|c|}
\hline & \multicolumn{2}{|c|}{ Población } & \multicolumn{2}{|c|}{$\begin{array}{c}\text { Migrantes laborales } \\
\text { a Estados Unidos }\end{array}$} \\
\hline & Total & 12 años y más & Enadid & Promig \\
\hline Región centro-occidente & 22.2 & 21.8 & 42.8 & 83.4 \\
\hline Aguascalientes & 0.9 & 0.9 & 1.4 & 0.0 \\
\hline Colima & 0.5 & 0.5 & 1.0 & 1.9 \\
\hline Guanajuato & 4.9 & 4.8 & 8.4 & 19.8 \\
\hline Jalisco & 6.6 & 6.5 & 12.6 & 13.6 \\
\hline Michoacán & 4.4 & 4.2 & 11.4 & 27.8 \\
\hline Nayarit & 1.0 & 1.0 & 1.4 & 9.2 \\
\hline San Luis Potosí & 2.4 & 2.4 & 2.3 & 5.2 \\
\hline Zacatecas & 1.5 & 1.5 & 4.3 & 5.9 \\
\hline Otros estados & 77.8 & 78.2 & 57.2 & 16.6 \\
\hline Total relativo & $100.0 \%$ & $100.0 \%$ & $100.0 \%$ & $100.0 \%$ \\
\hline Total absoluto (miles) & 85966 & 60218 & 2449 & 38 \\
\hline
\end{tabular}

Fuentes: Encuesta Nacional de la Dinámica Demográfica, 1992 y Proyecto sobre Migración Mexicana.

Entre los estados de la región histórica de la migración MéxicoEstados Unidos, los datos de Jalisco muestran una representación igual al interior del Promig y la Enadid (13.6 y $12.6 \%$, respectivamente). Una situación similar puede decirse que existe en los estados de Colima y Zacatecas. La ausencia de Aguascalientes no parece afectar seriamente la representación de la migración internacional mexicana en el Promig, pues su contribución a ésta es realmente pequeña según la información de la Enadid (1.4\%). El sesgo de los datos del Promig proviene sustancialmente de la sobrerrepresentación de la migración de Guanajuato y Michoacán. Mientras que los trabajadores internacionales del primer estado constituyen $8 \%$ del total nacional en la Enadid, éstos constituyen $20 \%$ del total de la muestra del Promig. En el caso de Michoacán, estas cifras son de 11 y $28 \%$ respectivamente. 
En resumen, la estrategia muestral del Promig ha producido una base de datos de migrantes laborales mexicanos con una gran concentración en la región centro-occidente del país, particular mente de los estados de Guanajuato y Michoacán. Sin embargo, es importante hacer notar que el Promig no fue diseñado con el objetivo de representar muestralmente la migración de todo México hacia Estados Unidos, por lo que la comparación es hasta cierto punto inadecuada. Hasta ahora únicamente se ha confirmado un hecho en sí mismo evidente: que el Promig contiene principalmente migrantes de la región histórica de la migración México-Estados Unidos. Una comparación más pertinente debe derivarse de contrastar el Promig y la Enadid en el interior de esta región. El cuadro 3 presenta información relevante para esta tarea.

\section{GUADRO 3}

Prevalencia de la migración mexicana hacia Estados Unidos en la región centro-occidente y entidades federativas seleccionadas

\begin{tabular}{|c|c|c|c|c|c|c|}
\hline & \multicolumn{3}{|c|}{ Migrantes laborales } & \multicolumn{3}{|c|}{ Prevalencia de la migración } \\
\hline & Enadid & Promig & Diferencia & Enadid & Promig & Diferencia \\
\hline Región centro-occidente & & & & $8.1 \%$ & $10.1 \%$ & \\
\hline Aguascalientes & $3.2 \%$ & $0.0 \%$ & -3.2 & 6.2 & - & - \\
\hline Colima & 2.0 & 2.3 & 0.3 & 6.6 & 8.9 & $2.3 \%$ \\
\hline Guanajuato & 19.1 & 23.8 & 4.7 & 7.1 & 9.4 & 2.3 \\
\hline Jalisco & 28.5 & 16.3 & -12.2 & 7.9 & 10.0 & 2.1 \\
\hline Michoacán & 25.8 & 33.3 & 7.5 & 11.1 & 12.7 & 1.6 \\
\hline Nayarit & 3.2 & 11.0 & 7.8 & 5.8 & 10.5 & 4.7 \\
\hline San Luis Potosí & 5.3 & 6.2 & 0.9 & 4.0 & 8.4 & 4.4 \\
\hline Zacatecas & 9.8 & 7.1 & 2.7 & 11.8 & 12.6 & 0.8 \\
\hline Total absoluto (miles) & 1083 & 32 & & 1083 & 32 & \\
\hline \multicolumn{7}{|l|}{ Estados seleccionados } \\
\hline Guanajuato & $21.6 \%$ & $24.8 \%$ & 3.2 & $7.1 \%$ & $9.4 \%$ & 2.3 \\
\hline Jalisco & 32.2 & 33.6 & 1.4 & 7.9 & 10.0 & 2.1 \\
\hline Michoacán & 29.2 & 21.7 & -7.5 & 11.1 & 12.7 & 1.6 \\
\hline San Luis Potosí & 6.0 & 12.7 & 6.2 & 4.0 & 8.4 & 4.4 \\
\hline Zacatecas & 11.0 & 7.7 & -3.3 & 11.8 & 12.6 & 0.8 \\
\hline Total absoluto (miles) & 956 & 27 & & 956 & 27 & \\
\hline
\end{tabular}

Fuentes: Encuesta Nacional de la Dinámica Demográfica, 1992 y Proyecto sobre Migración Mexicana. 
Si se consideran las diferencias en los diseños muestrales, el Promig logra una representación adecuada de la migración internacional al interior de la región centro-occidente, particularmente de sus principales entidades federativas: Guanajuato, Jalisco, Michoacán, San Luis Potosí y Zacatecas. El Promig hace una excelente labor en los casos de Colima, Guanajuato, San Luis Potosí y Zacatecas. En cada uno de estos estados, la diferencia absoluta entre los porcentajes de migrantes reportados por el Promig y la Enadid es menor a los cinco puntos. Jalisco, sin embargo, está subrepresentado por cerca de 12 puntos porcentuales, mientras que Michoacán y Nayarit muestran una situación contraria (diferencia positiva de casi 8 puntos en cada estado). Al seleccionar sólo los cinco principales estados de la región (parte inferior del cuadro 3), el rango de las diferencias entre las dos encuestas disminuye aún más.

Las discrepancias geográficas son de esperarse dadas las metodologías del Promig y la Enadid. Una comparación distinta surge de la información concerniente a la prevalencia de la migración temporal hacia Estados Unidos (el número total de migrantes relativo al número de personas de 12 años y más de edad). Según la Enadid, cerca de $4.1 \%$ de todos los mexicanos en este rango de edad han ido a ese país en calidad de migrantes laborales (este dato no se presenta en el cuadro); cifra que se dobla a $8.1 \%$ en el caso de la región centrooccidente. Según el Promig, sin embargo, la incidencia de la migración en esta región es de $10.1 \%$. Aun cuando las comunidades incluidas en el Promig hayan sido seleccionadas sin algún criterio en relación con el fenómeno migratorio México-Estados Unidos, esta base de datos reporta en términos relativos un mayor número de trabajadores internacionales que la Enadid. Como se verá más adelante, esta diferencia es mayormente resultado de una mejor enumeración de miembros ausentes del hogar en el Promig que en la Enadid.

Tal y como se muestra en la última columna del cuadro 3 , las diferencias en la prevalencia de migrantes laborales internacionales entre el Promig y la Enadid muestran un patrón regular. Por un lado, en todos los casos estas "tasas" de emigración son superiores en la orimera fuente que en la segunda. Por el otro, en la mayoría de los zasos la sobrestimación del Promig es en números absolutos menor a 2.5\%. Las excepciones son San Luis Potosí y Nayarit, donde la difeencia es de aproximadamente $4.5 \%$. En cualquier caso, el Promig sapta más migrantes que la Enadid, ya sea como producto de selec:ionar inadvertidamente comunidades con una incidencia mayor de 
movimientos temporales hacia Estados Unidos, o como resultado de enumerar de forma más adecuada a los miembros del hogar ausentes por estar en Estados Unidos.

Características de los migrantes laborales: comparaciones univariadas

El cuadro 4 presenta información sobre las características de los migrantes laborales identificados en el Promig y la Enadid. El análisis gira alrededor de dos comparaciones. Por un lado entre el total de migrantes laborales registrados en ambas fuentes, y por el otro entre aquellos que residen en los principales estados de la región tradicional de la migración México-Estados Unidos (Guanajuato, Jalisco, Michoacán, San Luis Potosí y Zacatecas).

Como los datos indican, el enfoque metodológico del Promig no produce una distribución representativa de migrantes por tamaño de localidad de residencia. En general, este proyecto sobrerrepresenta migrantes de comunidades de tamaño mediano, mientras que los migrantes en los extremos rural y urbano están subrepresentados. Según la Enadid, 31\% de todos los migrantes internacionales tienen como residencia comunidades con menos de 2500 habitantes. El Promig sólo cuenta con $5 \%$ de migrantes de origen rural. El Promig también discrepa de la Enadid en el porcentaje de mexicanos con experiencia laboral en Estados Unidos que viven en ciudades de 100 mil o más habitantes (25 y 41\%, respectivamente). Como se deriva de estas estadísticas, el Promig cuenta con una concentración muy alta de migrantes de comunidades con un rango de población de 20 mil a 100 mil habitantes (37\%). Este mismo patrón por tamaño de localidad puede observarse al contrastar la región tradicional de la migración mexicana hacia Estados Unidos. En consecuencia, la naturaleza del Promig ha llevado no sólo a una concentración significativa de sus microestudios en los estados de esta región en el conjunto del país, sino también en comunidades muy particulares al interior de éstos, principalmente lugares con una población de entre 2500 y 100 mil habitantes. 
CUADRO 4

Características sociales de los trabajadores mexicanos con experiencia laboral en Estados Unidos según el Proyecto sobre Migración Mexicana (Promig) y la Encuesta Nacional de la Dinámica Demográfica (Enadid)

\begin{tabular}{|c|c|c|c|c|c|}
\hline & \multicolumn{2}{|c|}{ Muestra total } & \multicolumn{2}{|c|}{$\begin{array}{c}\text { Muestra estados } \\
\text { seleccionados }\end{array}$} & \multirow{2}{*}{$\frac{\begin{array}{c}\text { Otros } \\
\text { estados }\end{array}}{\text { Promig }}$} \\
\hline & Promig & Enadid* & Promig & Enadid & \\
\hline \multicolumn{6}{|l|}{ Tamaño de localidad } \\
\hline Menos de 2500 habitantes & $5.0 \%$ & $30.8 \%$ & $7.0 \%$ & $38.7 \%$ & $25.4 \%$ \\
\hline $2500-19999$ & 33.1 & 20.1 & 33.1 & 23.9 & 17.4 \\
\hline $20000-99999$ & 37.0 & 7.9 & 48.5 & 9.5 & 6.8 \\
\hline 100000 y más & 24.8 & 41.3 & 11.4 & 27.8 & 50.3 \\
\hline \multicolumn{6}{|l|}{ Sexo } \\
\hline Hombres & $85.3 \%$ & $84.4 \%$ & $87.5 \%$ & $87.7 \%$ & $82.1 \%$ \\
\hline Mujeres & 14.7 & 15.6 & 12.5 & 12.3 & 17.9 \\
\hline \multicolumn{6}{|l|}{ Edad } \\
\hline Mediana & 39.0 & 37.0 & 38.0 & 37.0 & 38.0 \\
\hline Media & 40.9 & 40.8 & 40.7 & 40.2 & 41.2 \\
\hline Desviación estándar & 16.3 & 16.1 & 16.7 & 15.6 & 16.5 \\
\hline \multicolumn{6}{|l|}{ Relación con jefe(a) del hogar } \\
\hline Jefe(a) del hogar & $65.1 \%$ & $69.9 \%$ & $65.5 \%$ & $70.9 \%$ & $69.3 \%$ \\
\hline Cónyuge & 7.3 & 8.8 & 6.3 & 7.1 & 10.1 \\
\hline $\mathrm{Hijo}(\mathbf{a})$ & 26.1 & 15.6 & 27.0 & 17.0 & 14.7 \\
\hline Otra & 1.5 & 5.6 & 1.2 & 5.1 & 6.0 \\
\hline \multicolumn{6}{|l|}{ Estado marital } \\
\hline Actualmente casado(a) o unido (a) & $72.7 \%$ & $77.3 \%$ & $72.0 \%$ & $79.7 \%$ & $75.7 \%$ \\
\hline Soltero(a) & 22.5 & 15.3 & 23.7 & 15.0 & 15.5 \\
\hline Alguna vez casado(a) o unido(a) & 4.8 & 7.4 & 4.3 & 5.3 & 8.8 \\
\hline \multicolumn{6}{|l|}{ Años de Educación Formal } \\
\hline Menos de 6 años & $44.7 \%$ & $47.9 \%$ & $52.4 \%$ & $56.1 \%$ & $42.3 \%$ \\
\hline $6-8$ años & 30.1 & 28.2 & 26.2 & 27.4 & 28.7 \\
\hline 9-11 años & 13.1 & 16.7 & 13.1 & 12.6 & 19.3 \\
\hline $12+$ años & 12.1 & 7.1 & 8.2 & 4.0 & 9.2 \\
\hline Mediana & 6.0 & 6.0 & 5.0 & 4.0 & 6.0 \\
\hline Media & 5.6 & 5.4 & 5.0 & 4.6 & 5.9 \\
\hline Desviación estándar & 4.2 & 3.8 & 4.0 & 3.6 & 3.9 \\
\hline \multicolumn{6}{|l|}{ Duración del último viaje a EUA } \\
\hline Mediana & 8.0 & 7.0 & 7.0 & 7.0 & 7.0 \\
\hline Media & 21.9 & 20.1 & 21.2 & 19.8 & 20.3 \\
\hline Desviación estándar & 46.3 & 40.4 & 46.5 & 36.7 & 42.8 \\
\hline \multicolumn{6}{|l|}{ Número de casos } \\
\hline Expandidos (miles) & 38 & 2372 & 27 & 956 & 1416 \\
\hline Sin Expandir & 4255 & 8765 & 3948 & 2608 & 6157 \\
\hline
\end{tabular}


Contra lo que se pudiera esperar, este sesgo del proyecto no produce marcadas diferencias en las características individuales de los migrantes en contraste con la muestra representativa de la Enadid, tanto para el caso de la misma región centro-occidente como del conijunto nacional. Esto permite afirmar que los procesos sociales y económicos subyacentes a la migración laboral México-Estados Unidos, parecen operar con una lógica poco diferenciadora según el tamaño de la localidad de origen. Esta hipótesis puede argumentarse como cierta por lo menos para la región con mayor tradición migratoria internacional (la mejor representada en el Promig).

El perfil de los migrantes resulta sorprendentemente similar en ambas fuentes no obstante sus diferencias metodológicas. Por ejemplo, la diferencia absoluta en la distribución por sexo es de tan sólo un punto porcentual. Esta variación es aún menor cuando se compara con los estados de la región centro-occidente, donde ambas fuentes reportan que $88 \%$ de los migrantes laborales internacionales son hombres. Si bien estas diferencias son estadísticamente significativas dado el gran tamaño de las muestras, ellas son obviamente de una importancia irrelevante.

El Promig y la Enadid producen también estructuras por edad muy similares. Aunque la edad mediana difiere por dos años en la comparación total y en un año entre los estados seleccionados, la edad media y las desviaciones estándares son prácticamente las mismas: 40.9 en la primera base de datos y 40.8 en la segunda, respectivamente ( 40.7 y 40.2 en la región centro-occidente). Ambas fuentes de información son entonces consistentes al mostrar que la población mexicana con experiencia migratoria y laboral en Estados Unidos tiene un promedio de edad de 41 años y una edad mediana de entre 37-39 años, indicando con ello la existencia de una variación significativa (desviación estándar de aproximadamente 16 años en ambas bases de datos). Una vez más las discrepancias son estadísticamente significativas pero de una importancia poco sustantiva.

Las diferencias en el nivel educativo de los migrantes son también muy pequeñas. Al considerar el conjunto de la población con experiencia laboral en Estados Unidos, $45 \%$ de los migrantes internacionales captados por el Promig tienen una educación menor a la primaria completa, en contraste con $48 \%$ registrado por la Enadid. Los porcentajes de trabajadores internacionales con una escolaridad de 6 a 8 años de instrucción formal son también muy cercanos. Las principales disimilitudes entre estas encuestas aparecen en los nive- 
les de educación más altos, lo cual es muy probablemente reflejo de la sobrerrepresentación de ciudades de tamaño mediano en el Promig, y de la subestimación de las localidades más rurales. Mientras que $12 \%$ de los migrantes listados en el Promig tiene 12 o más años de educación formal, sólo $7 \%$ de los de la Enadid cumplen con esta condición.

No obstante las variaciones en los extremos de la distribución de esta variable, las medias son prácticamente iguales (5.6 en el Promig y 5.4 en la Enadid), y las medianas son exactamente del mismo valor (6 años). El mismo patrón de comparación prevalece entre los migrantes de la región centro-occidente, aunque las medias difieren más (5.0 en el Promig y 4.6 en la Enadid). En cualquier caso, ambas bases de datos producen un perfil educativo de los migrantes internacionales muy parecido. Una mayoría en la región histórica, y cerca de la mitad de los migrantes en el total del país, tienen una educación menor a la primaria terminada, con un promedio de educación de 4-5 años en el primer conjunto y de 5-6 en el segundo.

Probablemente la estadística más sorprendente en la contrastación del Promig y la Enadid es con respecto a la duración del viaje más reciente a Estados Unidos. Esta variable, la única relacionada con la experiencia migratoria en sí misma, fue determinada únicamente para los mexicanos que ya habían regresado al país. En el total de las muestras, la duración promedio de estos viajes fue de 21.9 meses en el Promig y de 20.1 meses según la Enadid. Las medianas de sus distribuciones fueron de 8 y 7 meses respectivamente, indicando con ello la existencia de una gran variación en la duración de los viajes. Entre los migrantes de la región histórica de la migración hacia Estados Unidos estas estadísticas son aún más cercanas: la duración mediana es exactamente la misma en ambas bases de datos ( 7 meses), y la duración promedio es de 21.2 meses en el Promig y de 19.8 meses en la Enadid. Si bien la duración promedio de las migraciones temporales es siempre más alta en la primera fuente que en la segunda, las diferencias son realmente pequeñas para un factor que muestra una gran variación.

La marcada correspondencia de la duración de los viajes es ilustrada de manera más nítida en la gráfica 1 , la cual muestra la distribución acumulada de esta variable según el Promig y la Enadid. La coincidencia es realmente extraordinaria, particularmente la abrupta subida que ambas bases de datos muestran en el intervalo de 24 a 25 meses. Cualesquiera que sean los errores de declaración en lo que 
respecta a la duración de las migraciones a Estados Unidos, éstos son consistentes en ambas fuentes.

La distribución de la extensión de los viajes es generalmente más variable en el Promig (véanse las desviaciones estándar), lo cual puede ser un refilejo de la forma en que esta variable es operacionalizada en cada encuesta. En la Enadid se conoce el mes y el año de salida y llegada de los migrantes, por lo que la duración se calcula por sustracción. En cambio, en el Promig los migrantes proporcionan su propio cálculo de la duración del viaje. No es por lo tanto ninguna sorpresa que el cálculo establecido por el entrevistado en este último proyecto sobre la duración de su último viaje a Estados Unidos muestre un mayor error en comparación con el cálculo directo que es posible hacer con los datos de la Enadid. A pesar de esta gran variación, las estadísticas del Promig no parecen estar sesgadas de manera sistematizada, tal y como lo indican las estadísticas descriptivas anteriormente examinadas.

En contraste con las variables anteriores, la información sobre la relación del parentesco con elijefe del hogar y el estado marital de los migrantes internacionales muestra una mayor diferenciación. Comparado con los migrantes identificados en la Enadid, el Promig reporta menosijefes(as) de hogares y más hijos(as) delijefe(a). Relacionado estrechamente con esta situación, sólo $73 \%$ de los migrantes en el Promig estaban casados o unidos en el momento de la encuesta ( $77 \%$ en la Enadid), y $23 \%$ nunca se había casado o unido ( $15 \%$ en la Enadid). Estas diferencias son significativas tanto en términos estadísticos como sustantivos, y el mismo patrón se presenta al comparar a los estados de la región centro-occidente.

Estas discrepancias pueden ser interpretadas de dos maneras. La primera, que la forma de selección de las comunidades en el Promig produce una muestra sesgada que no refileja de manera precisa las características de los migrantes mexicanos con experiencia laboral en Estados Unidos. Sin embargo, en esta situación uno tendría que explicar por qué el Promig coincide de forma tan marcada con la Enadid en relación con las características de sexo, edad, educación y duración de la migración. Si bien el sesgo no necesita ser consistente entre variables, en general es de esperarse que éste se presente de una manera más uniforme.

La segunda posible explicación es que el Promig realiza una mejor labor identificando hijos(as) solteros(as) del jefe del hogar que están trabajando en Estados Unidos, y por lo tanto reportando más mi- 


\section{GRÁFICA 1}

Duración en meses de la última migración laboral a EUA de los trabajadores internacionales residentes en los principales estados de la región centro-occidente de México. Porcentaje acumulado

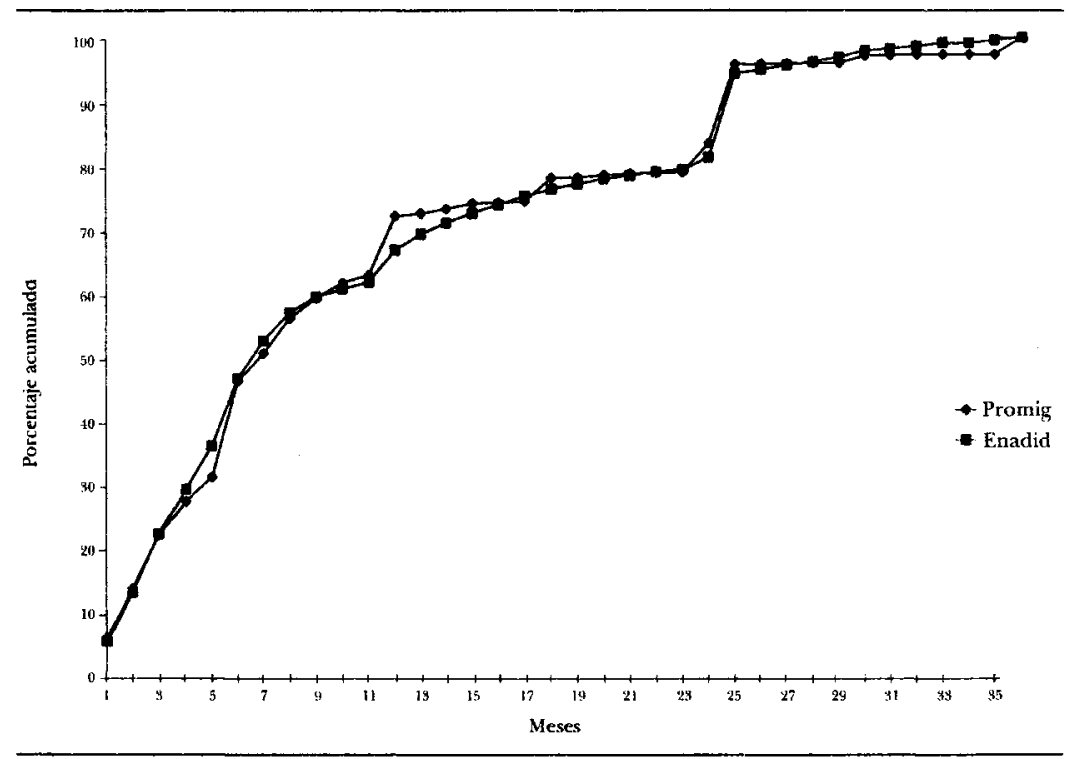

grantes en estas categorías que la Enadid. Como una encuesta demográfica formal, la Enadid utiliza preguntas estandarizadas no sólo para listar a los residentes habituales del hogar, sino también para determinar quiénes son migrantes internacionales. La Enadid no incluye ninguna pregunta específica para detectar que un miembro del hogar no haya sido declarado como residente habitual por estar ausente debido a que labora temporalmente en Estados Unidos. Un migrante internacional ausente es únicamente reportado como residente habitual en el caso de que el informante lo haya considerado así. El Promig, a diferencia de la Enadid, fue diseñado explícitamente para el estudio del proceso de la migración circular entre México y Estados Unidos, por lo que los encuestadores realizan esfuerzos especiales para enumerar a miembros del hogar que han estado trabajando por un largo tiempo en Estados Unidos. El hecho de que el Promig reporta más hijos(as) solteros(as) y una mayor duración pro- 
medio de los viajes, sugiere que los procedimientos para captar a estos residentes del hogar son más eficientes en el Promig que en la Enadid. Esta interpretación recibe apoyo adicional del hecho de que el Promig reporta un porcentaje mayor de migrantes internacionales ausentes que la Enadid ( 10 y $6 \%$, respectivamente). En este sentido, las discrepancias entre estas fuentes en lo que respecta a relación de parentesco y estado marital, indican la mayor eficiencia del Promig para identificar migrantes internacionales sin menoscabo en la medición de otras características de los migrantes.

Características de los migrantes laborales: un ejercicio multivariado

Las comparaciones univariadas sugieren que, una vez que las diferencias metodológicas entre el Promig y la Enadid son tomadas en consideración, sus resultados no producen diferencias sustantivas en el perfil sociodemográfico de los migrantes con experiencia laboral en Estados Unidos. Pero, ¿qué tanto esta situación puede ser un resultado fortuito al estar únicamente comparando estadísticas univariadas? ¿Es posible que estas similitudes se sostengan en un contexto de análisis estadístico más demandante? El objetivo de esta sección es precisamente ampliar el contraste entre estas dos fuentes de información a una situación estadística multivariada; más específicamente, al examen de las propensiones de migrar a Estados Unidos. En años recientes la información del Promig ha sido utilizada para producir una variedad de modelos estadísticos con el objetivo de predecir las probabilidades de migrar a Estados Unidos (Massey et al., 1987; Massey, 1987; Delechat, 1994, 1995; Zahniser, 1996; Massey y Espinosa, 1997). ¿Qué tanto hubieran variado los resultados y conclusiones de estas investigaciones de haber producido estos modelos con información representativa de la región estudiada por el Promig, o incluso representantiva del contexto nacional?

El cuadro 5 busca contestar a esta pregunta calculando modelos de regresión logística para predecir los logaritmos de los momios ( $\log$-odds) de migrar de Estados Unidos por motivos laborales en función de algunas variables independientes, utilizando para ello tanto información de la Enadid como del Promig. Con base en el trabajo de Massey et al. (1987) y de otros investigadores que han estudiado la migración a Estados Unidos con información de corte transversal, el 
análisis se centra únicamente en los movimientos internacionales que tuvieron lugar durante los tres años previos al levantamiento de la encuesta. Este periodo de referencia produce un número más adecuado de casos y una mejor distribución de la variable dependiente que uno de tan sólo un año, permitiendo con ello la estimación de resultados más estables y confiables.

Para el análisis se seleccionaron personas de 15 años y más de edad. La condición migratoria se codificó como 1 cuando alguna persona viajó a Estados Unidos durante este periodo de tres años, y 0 cuando no ocurrió así. Este resultado binario se examinó en función de la edad, sexo, relación de parentesco con el jefe del hogar, educación, tamaño y tipo de hogar, dependencia de niños menores de 12 años en el hogar, y el tamaño de la localidad. Se estimó el mismo modelo de regresión logística con los datos de la Enadid y el Promig, con la diferencia de que en esta última se controló además por el año de la encuesta (los coeficientes no se muestran por razones de espacio). Los resultados de este ejercicio se presentan en las primeras cuatro columnas del cuadro. La comparación sistemática de los coeficientes permite:juzgar el grado de sesgo de los modelos estimados con los datos del Promig.

Los resultados basados en la Enadid sugieren que la propensión a migrar alcanza su punto más alto en las edades 25-34 años, que los hombres y los jefes de hogares son más propensos a migrar internacionalmente que las mujeres y otros miembros del hogar (respectivamente), que existe una selectividad negativa de los migrantes a Estados Unidos según la educación, y que la migración al norte es más probable que tenga su origen en comunidades rurales muy pequeñas (menos de 2500 habitantes). El resto de variables incluidas en el modelo no fueron estadísticamente significativas.

Los coeficientes estimados con los datos del Promig describen esencialmente la misma historia. Si bien el tamaño relativo de los coeficientes no es el mismo como resultado de no contar con tamaños de muestra y distribuciones de variables semejantes, sólo con una excepción los resultados producen un patrón similar de coeficientes estadísticamente significativos. Según ambas encuestas, la propensión más alta de migrar ocurre entre la población de 25-34 años, con menor nivel educativo, masculina, jefe(a) de hogar y que reside en comunidades extremadamente rurales. La única diferencia entre las dos ecuaciones es con respecto al tamaño de la familia; variable que tiene un efecto positivo significativo en la probabilidad de 


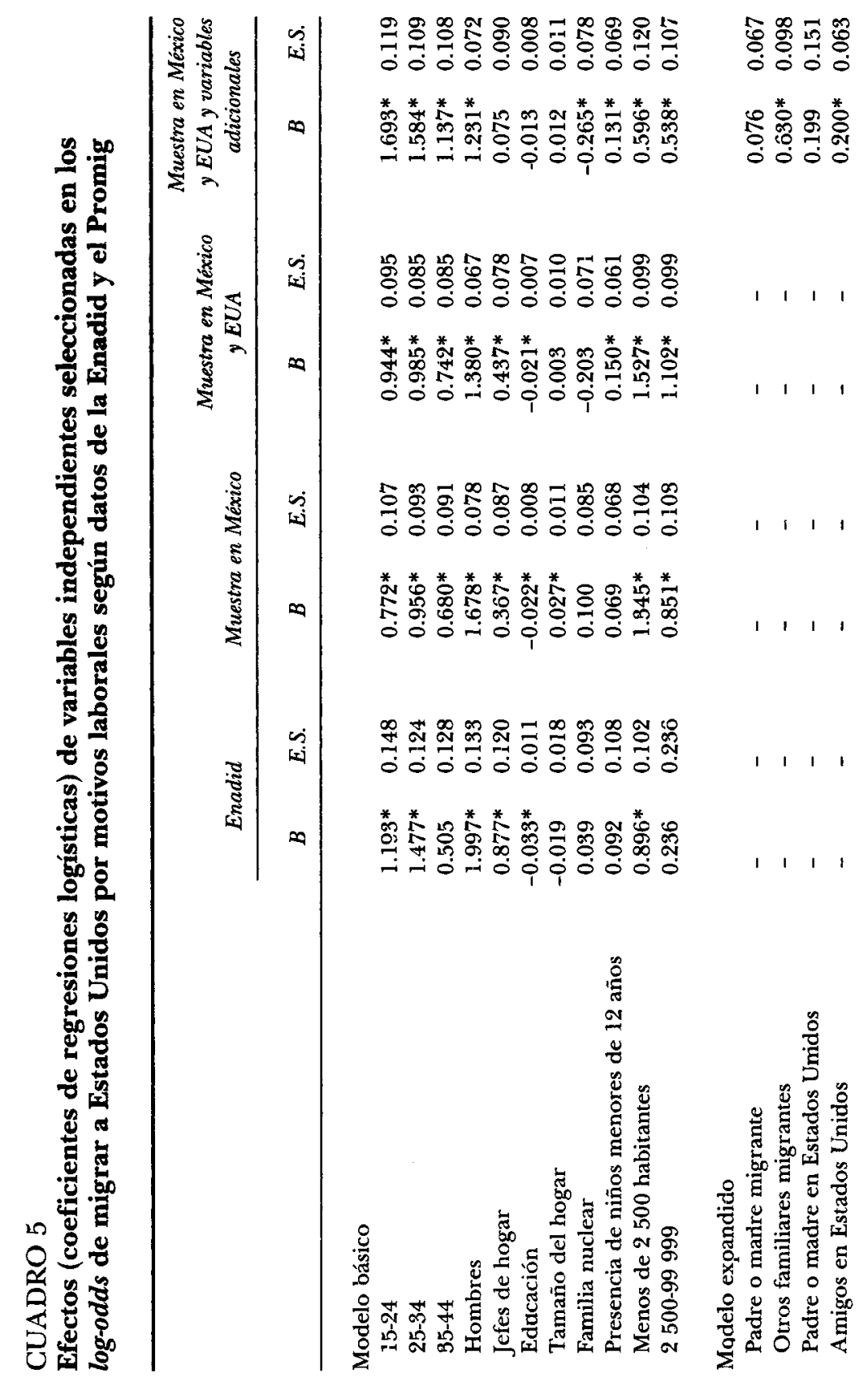




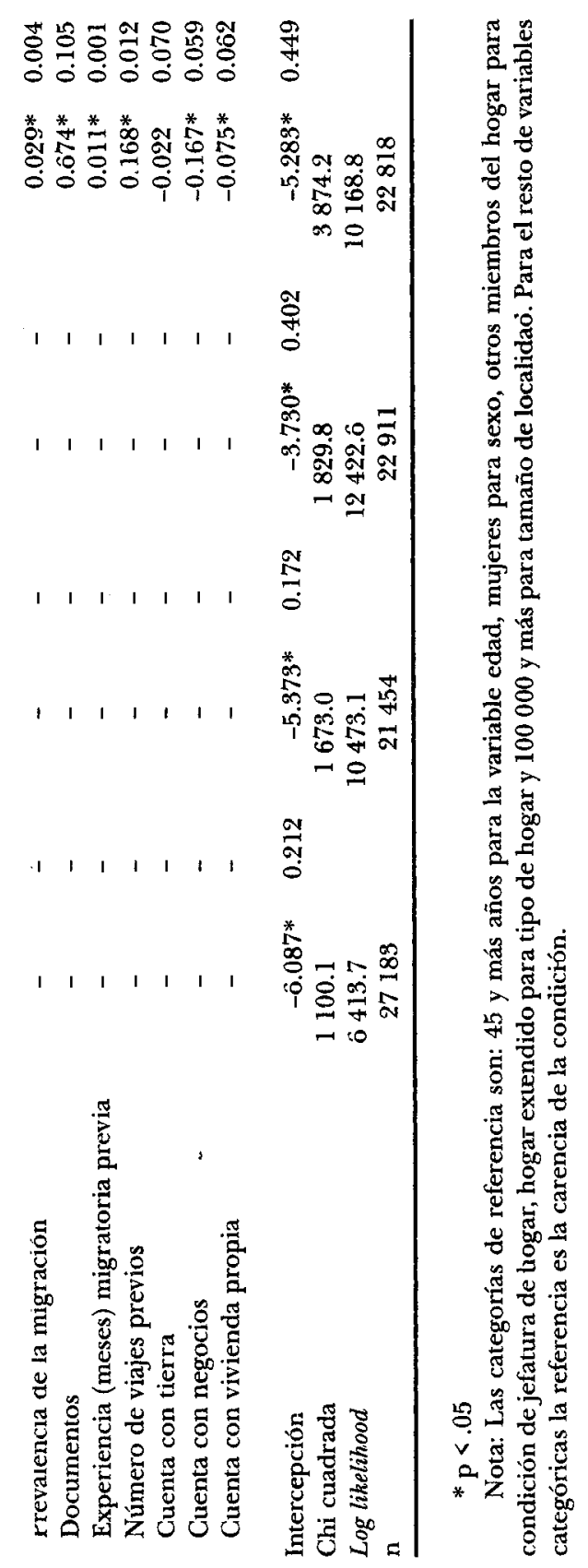


migrar cuando el modelo es calculado con los datos del Promig. Esta variable muestra un efecto negativo pero no significativo en el caso de la Enadid. Este contraste puede estar relacionado con la subenumeración de hijos(as) que trabajan en Estados Unidos en esta última encuesta, la cual probablemente esté produciendo una subestimación tanto del tamaño de la familia como en las propensiones de emigrar.

En cualquier caso, la estrategia de muestreo de comunidades del Promig no parece introducir un sesgo significativo en la predicción de la migración temporal de mexicanos hacia Estados Unidos. Es claro que el sesgo que resulta del uso de métodos de muestreo no probabilísticos en un nivel agregado de la región centro-occidente no es la única posible fuente potencial de error en un diseño de investigación de encuestas. El sesgo de selección representa otro problema importante en el análisis multivariado. De esta manera la validez de la Enadid disminuye por el hecho de que no incluye a miembros del hogar que se han establecido de forma más permanente en Estados Unidos. En contraste, el Promig realiza esfuerzos especiales para entrevistar a personas en algunas áreas de destino de los migrantes en Estados Unidos.

Con el fin de determinar en qué medida este sesgo de selección afecta los resultados de la Enadid y de otras encuestas realizadas exclusivamente en México, la ecuación de regresión con los datos del Promig fue calculada nuevamente agregando las muestras de migrantes que residen en Estados Unidos. La regresión incluye a todos los migrantes que vivían en México tres años antes de la encuesta y viajaron a Estados Unidos previamente al levantamiento de la misma.

La inclusión de los residentes permanentes en Estados Unidos cambia los patrones de los coeficientes de manera importante. Primero, el diferencial por edad se modera considerablemente. Los mexicanos con una edad de 15-24 años tienen ahora casi la misma probabilidad de emigrar que los que cuentan con 25-34 años, y entre aquellos que tienen 35-44 años la propensión a migrar es un poco más baja. Segundo, la dependencia familiar indicada por la presencia de niños menores de 12 años aparece en la nueva ecuación como un factor positivo en las posibilidades de migración a Estados Unidos, mientras que el efecto del tamaño de familia en sí mismo desaparece. La exclusión de hogares que se han establecido al norte de la frontera no permite observar el efecto de la dependencia familiar en la migración internacional, efecto previamente reportado en Massey et al. (1987). Más allá de los efectos de edad y dependencia 
familiar, los patrones de relación de las variables independientes son similares a los producidos con los datos de la Enadid.

Un último sesgo potencial emana de los errores de especificación, esto es, de las dificultades de estimar un modelo de migración excluyendo factores teóricamente relevantes. En este caso, el número tan pequeño de antecedentes individuales y familiares con los cuales permite trabajar la Enadid, implica una alta probabilidad de que los coeficientes estimados estén sesgados debido a la omisión de factores cruciales en la decisión de migrar a Estados Unidos, tales como el acceso al capital social que proveen las redes de migrantes (Taylor, 1986 y 1987; Delechat, 1993, 1994 y 1995; Zahniser, 1996; Espinosa y Massey, 1998; Massey y Espinoza, 1997). El último ejercicio de evaluación agrega a nuestros modelos estadísticos importantes antecedentes de los jefes de hogar disponibles en la base de datos del Promig: la condición migrante internacional de sus padres, la condición de migración internacional de otros parientes, si alguno de sus padres reside en Estados Unidos al momento de la encuesta, si cuenta o no con amistades al norte de la frontera, si posee o no documentos legales para cruzar la frontera, la experiencia acumulada y el número de viajes realizados a Estados Unidos, y la condición de propiedades (vivienda, tierra y negocios).

Los resultados presentados en las dos últimas columnas demuestran claramente los problemas de especificación de encuestas generales como la Enadid, las cuales omiten variables relevantes para el entendimiento de problemáticas sociales particulares. Como ha sido demostrado con mayor detenimiento en otros trabajos, factores asociados con el capital social y el capital humano relacionado específicamente con la migración son altamente significativos en la predicción de la emigración hacia Estados Unidos. Las personas con mayor número de viajes y más acumulación de experiencia en el país del norte, y con documentos para viajar legalmente a Estados Unidos son, para ninguna sorpresa, considerablemente más probables de migrar a ese país que personas sin tales recursos. Igualmente, individuos con acceso directo a capital social (familiares con experiencia en Estados Unidos, amistades que viven en Estados Unidos, y residen en comunidades donde la prevalencia de migración internacional es alta) tienen una mayor propensión a viajar a Estados Unidos que aquellos que carecen de él. En general, la posesión de capital físico en México (tierras, negocios o viviendas) reduce las probabilidades de un movimiento internacional. 
La inclusión de estas variables reduce aún más el efecto diferencial por edad, por un lado, y elimina los efectos significativos atribuibles a la jefatura de hogar y a la educación, por el otro. Además, los resultados revelan el efecto previamente suprimido de la estructura familiar: los individuos que viven en hogares nucleares tienen significativamente menos probabilidades de migrar a Estados Unidos que aquellos que viven en hogares extendidos. Como es de esperarse, estos últimos están en una mejor posición que los anteriores de diversificar sus estrategias de sobrevivencia y reproducción mediante la migración laboral internacional.

Así pues, los sesgos de selección y los errores de especificación afectan las estimaciones basadas en los datos de la Enadid. Al concentrar la atención en un modelo estadístico que sólo incluye las variables proporcionadas por la Enadid, se obtienen resultados muy similares entre esta fuente y el Promig. Sin embargo, un perfil muy distinto de la migración emerge al incluir en subsecuentes modelos a migrantes mexicanos que se han establecido de forma más permanente en Estados Unidos, y al agregar otros factores de relevancia teórica para la comprensión de la migración internacional. De trabajar únicamente con la información de la Enadid (o la muestra comparable del Promig), se concluiría que las propensiones a emigrar están estrechamente relacionadas con la edad, el sexo, la relación de parentesco y la educación. Una vez que el sesgo de selección y los errores de especificación son corregidos al incluir más casos y variables, la información del Promig muestra que la decisión de migrar internacionalmente no está tan influida por la edad, la relación de parentesco y la educación, mientras que la estructura familiar, la dependencia de menores, el capital humano específico a la migración y el acceso a capital social aparecen como factores asociados de manera significativa con la migración laboral a Estados Unidos.

\section{Consideraciones finales}

La comparación de la información del Promig y la Enadid ha permitido examinar, para el caso específico de la migración internacional, los alcances de investigaciones cuya búsqueda de una mayor especificidad en el análisis de fenómenos sociales implica metodológicamente el estudio sistemático de comunidades, en contraposición a aquéllas cuyos afanes de generalización conllevan la selección de grandes agre- 
gados poblacionales y el sacrificio de la profundidad analítica. Más específicamente, se intentó examinar las principales fuentes de error originadas por las particularidades metodológicas del Promig, al no recolectar muestras representativas de la región con mayor tradición migratoria hacia Estados Unidos.

$\mathrm{Ni}$ el análisis descriptivo de las características de la población mexicana con experiencia laboral en Estados Unidos, ni los análisis multivariados de las propensiones a viajar a dicho país por motivos laborales, arrajaron evidencias sobre la existencia de sesgos significativos en los alcances de generalización del Promig. Si bien el Promig reporta una sobrerrepresentación de población en ciudades de tamaño medio, y una mayor prevalencia de migración internacional como resultado de captar mejor migrantes ausentes solteros, con mayor nivel educativo y que no son jefes del hogar, las discrepancias son mínimas al comparar la composición por edad y sexo de los migrantes y la duración de los viajes a Estados Unidos. La sorprendente correspondencia entre la Enadid y el Promig es confirmada por los resultados multivariados prediciendo las propensiones a migrar a Estados Unidos para trabajar o buscar trabajo. Más aún, se ha podido mostrar empíricamente los sesgos en que incurren encuestas tradicionales como Enadid por muestrear selectivamente sólo migrantes internacionales residentes en México, y por no especificar dimensiones teóricas relevantes para la comprensión del proceso de la migración México-Estados Unidos.

El trabajo empírico aquí presentado sugiere dos conclusiones no necesariamente excluyentes entre sí. Primera, que la Enadid y el Promig muestren una gran correspondencia en el perfil de los migrantes internacionales, a pesar de sus diferentes distribuciones poblacionales según tamaño de localidad, indica la existencia de una gran homogeneidad en el interior de la región centro-occidente en lo que respecta al comportamiento del fenómeno migratorio México-Estados Unidos. Segunda, que los estudios basados en el análisis sistemático de comunidades pueden ser exitosos en la generalización de sus resultados para una región determinada, a pesar de no recolectar muestras probabilísticas representativas de la misma, ofreciendo además la ventaja de abordar el estudio de una problemática social con una mayor profundidad analítica. Esta última conclusión podrá ser evaluada con más detalle en un futuro próximo, al poder comparar y evaluar los resultados del Promig en cuatro comunidades de Tijuana estudiadas durante el presente año, con los resultados de la Encuestra Retros- 
pectiva sobre Migración y Empleo en Estados Unidos (ERMEU) en esta ciudad fronteriza. La ERMEU es un proyecto conjunto entre la Universidad de Pennsylvania y el Instituto Nacional de Estadística Geografía e Informática, con el fin de extender el trabajo del Promig a las principales áreas urbanas del país.

\section{Bibliografía}

Bean, Frank D., Thomas J. Espenshade, Michael J. White y Robert F. Dymowksi (1990), "Post-IRCA Changes in the Volume and Composition of Undocumented Migration to the United States: An Assessment Based on Apprehensions Data", en F. Bean, B. Edmonston y J. Passel (eds.), Undocumented Migration to the United States: IRCA and the Experience of the 1980s, Washington, Urban Institute.

Borjas, George J. (1985), "Assimilation, Changes in Cohort Quality, and the Earnings of Immigrants", Journal of Labor Economics, vol. 3, pp. 463489.

(1987), "Self-Selection and the Earnings of Immigrants", American Economic Review, vol. 3, pp. 531-553.

(1991), "Immigration and Self-Selection", en J. Abowdm y R. Freeman (eds.), Immigration, Trade, and the Labor Market, Chicago, University of Chicago Press.

Bustamante, Jorge (1989), "Medición del flujo de inmigrantes indocumentados", en J. Bustamante y W. Cornelius (coords.), Flujos migratorios mexicanos hacia Estados Unidos, México, Fondo de Cultura Económica.

(1998), "Generalizing and Sampling", Social Science Quarterly, vol. 79, núm. 1, pp. 21-22.

Colef (El Colegio de la Frontera Norte), Conapo (Consejo Nacional de Población) y STPS (Secretaría de Trabajo y Previsión Social) (1994), "Encuesta sobre Migración en la Frontera Norte. Síntesis ejecutiva", Tijuana, El Colegio de la Frontera Norte (mimeo.).

Conapo (Consejo Nacional de Población) (1986), Encuesta de la Frontera a Trabajadores Indocumentados Devueltos por las Autoridades de los Estados Unidos de América: diciembre de 1994, México, Conapo.

Corona , Rodolfo (1990), "La medición del fenómeno migratorio en el censo de población de 1990", Frontera Norte, vol. 2, núm. 3, pp. 5-30.

(1991), "Confiabilidad de los resultados preliminares del XI Censo General de Población y Vivienda de 1990", Estudios Demográficos y Urbanos, vol. 6, núm. 1, pp. 33-68.

(1993), "Migración permanente interestatal e internacional, 19501990", Comercio Exterior, vol. 43, núm. 8, pp. 750-773.

Dagodag, W. Tim (1975), "Source Regions and Composition of Illegal Mexi- 
can Immigration to California" International Migration Review, vol. 9, pp. 499-511.

Davidson, C.A. (1981), "Characteristics of Deportable Aliens Located in the Interior of the United States", trabajo presentado en la Reunión Anual de la Asociación de Población de Estados Unidos, Washington (mimeo.).

Delechat, Corinne C. (1993), "Household Labor Supply and Migration Decisions: The Case of Mexican Labor Migration", trabajo presentado en la Binational Conference on Mexico-U.S. Migration, Chicago, Center for Latin American Studies, Universidad de Chicago (mimeo.).

(1994), "Mexican Labor Migration to the United States: Determinants, Labor Market Outcomes, and Dynamics", tesis de doctorado, Departamento de Economía, Universidad de Georgetown.

(1995), "Selectivity, English Language Proficiency, and the Earnings of Mexican Migrants in the United States", documento de trabajo, San Diego, Center for U.S.-Mexican Studies, Universidad de California.

Durand, Jorge, D. Massey y R. Zenteno (1998), "Reconsidering the 'Changing Character' of Mexican Migration to the United States", trabajo presentado en la Reunión Anual de la Asociación de Población de Estados Unidos, Chicago (mimeo.).

, Emilio Parrado y Douglas S. Massey (1995), "Migradollars and Development: A Reconsideration of the Mexican Case", International Migration Review, vol. 30, pp. 423-444.

Espenshade, Thomas J. (1990), "Undocumented Migration to the United States: Evidence from a Repeated Trials Model", en F. Bean, B. Edmonston y J. Passel (eds.), Undocumented Migration to the United States: IRCA and the Experience of the 1980s, Washington, Urban Institute.

(1994), "Does the Threat of Apprehension Deter Undocumented U.S. Immigration?", Population and Development Review, vol. 20, pp. 871-892. y Dolores Acevedo (1995), "Migrant Cohort Size, Enforcement Effort, and the Apprehension of Undocumented Aliens", Population Research and Policy Review, vol. 14, pp. 145-172.

Espinosa, Kristin y Douglas S. Massey (1998), "Undocumented Migration and the Quantity and Quality of Social Capital", Soziale Welt, vol. 12, pp. 141-162.

Fix, Michael y Jeffrey S. Passel (1994), Immigration and Immigrants: Setting the Record Straight, Washington, Urban Institute.

Gamio, Manuel (1930), Mexican Migration to the United States, Chicago, University of Chicago Press.

Goodman, Leo (1961), "Snowball Sampling", Annals of Mathematical Statistics, vol. 32, pp. 117-151.

Hill, Kenneth (1985), "Indirect Approaches to Assessing Stocks and Flows of Migrants", en D. Levine, K. Hill y R. Warren (eds.), Immigration Statistics: A Story of Neglect, Washington, National Academy Press. 
INEGI (1994), Encuesta Nacional de la Dinámica Demográfica, 1992. Metodología y tabulados, Aguascalientes, INEGI.

Jasso, Guillermina y Mark R. Rosenzweig (1982), "Estimating the Emigration

Rates of Legal Immigrants Using Administrative and Survey Data: The 1971 Cohort of Immigrants to the United States", Demography, vol. 19, pp. 279-290.

y Mark R. Rosenzweig (1990), The New Chosen People: Immigrants in the United States, Nueva York, Sage.

Jones, Richard C. (1988), "Micro Source Regions of Mexican Undocumented Migration", National Geographic Research, vol. 4, pp. 11-22.

Keely, Charles B. y B. Tran (1989), "Remittances from Labor Migration: Evaluations, Performance and Implications", International Migration Review, vol. 23, pp. 500-525.

Lindstrom, David P. y Douglas S. Massey (1994), "Selective Emigration, Cohort Quality, and Models of Immigrant Assimilation", Social Science Research, vol. 23, pp. 315-349.

Lozano Ascencio, Fernando (1993), "Bringing it Back Home: Remittances to Mexico from Migrant Workers in the United States", Monograph Series, 37, San Diego, Center for U.S.-Mexican Studies, Universidad de California.

y Judi L. McClellan (1997), "Immigration, Settlement in the United States, and Remittances: Evidence from the Mexican Case", trabajo presentado en el Encuentro Anual de la Asociación de Población de Estados Unidos, Washington, 27 al 29 de marzo (mimeo.).

Massey, Douglas S., Rafael Alarcón, Jorge Durand y Humberto González (1987), Return to Aztlan: The Social Process of International Migration from Western Mexico, Berkeley-Los Ángeles, University of California Press. (1987), "The Ethnosurvey in Theory and Practice", International Migration Review, vol. 21, pp. 1498-1522.

__ (1994), "The Methodology of an Ethnosurvey", en D. Bogue (ed.), Readings in the Methodology of Population Research, Nueva York, United Nations Fund for Population Activities.

(1998), "When Surveys Fail: An Alternative Approach to Studying Illegal Migration", en A. Stone (ed.), The Science of the Self-Report: Implications for Research and Practice, Nueva York, Erlbaum Press (en prensa). y Kristin E. Espinosa (1997), "What's Driving Mexico-U.S. Migration? A Theoretical, Empirical and Policy Analysis", American Journal of Sociology, vol. 102, pp. 939-999.

y Emilio A. Parrado (1994), "Migradollars: The Remittances and Savings of Mexican Migrants to the United States", Population Research and Policy Review, vol. 13, pp. 3-30.

North, David y Marion F. Houston (1976), The Characteristics and Role of Illegal Aliens in the U.S. Labor Market: The U.S. Bracero Experience, Washington, Litton. 
Passel, Jeffrey S. (1995), "Illegal Immigration: How Big a Problem?", trabajo presentado en la Conference on Latin American Migration: The Foreign Policy Dimension, Washington, U.S. Department of State, 17 de marzo (mimeo.).

y Barry Edmonston (1994), "Immigration and Race: Recent Trends in Immigration to the United States", en B. Edmonston y J. Passel (eds.), Immigration and Ethnicity: The Integration of America's Newest Arrivals, Washington, Urban Institute.

Pefeum (1995), Programa Nacional de Población 1995-2000, México, Poder Ejecutivo Federal de los Estados Unidos Mexicanos.

Portes, Alejandro (1979), "Illegal Immigration and the International System, Lessons from Recent Legal Mexican Immigrants to the United States", Social Problems, vol. 26, pp. 425-438.

Taylor, J. Edward (1986), "Differential Migration, Networks, Information and Risk", en O. Stark (ed.), Migration Theory, Human Capital and Development, Greenwich, JAI Press.

(1987), "Undocumented Mexico-U.S. Migration and the Returns to Households in Rural Mexico", American Journal of Agricultural Economics, vol. 69 , pp. 626-638.

U.S. Immigration and Naturalization Service (1990), Immigration Reform and Control Act: Report on the Legalized Alien Population, Washington, U.S. Government Printing Office. (1992), 1991 Statistical Yearbook of the Immigration and Naturalization Service, Washington, U.S. Government Printing Office. (1996), 1995 Statistical Yearbook of the Immigration and Naturalization Service, Washington, U.S. Government Printing Office.

Warren, Robert (1995), "Estimates of the Undocumented Immigrant Population Residing in the United States by Country of Origin and State of Residence, October 1992", trabajo presentado en el Encuentro Anual de la Asociación de Población de Estados Unidos, San Francisco (mimeo.).

y Ellen P. Kraly (1985), "The Elusive Exodus: Emigration from the United States", Population Trends and Public Policy, núm. 8, Washington, Population Reference Bureau.

White, Michael J., Frank D. Bean y Thomas J. Espenshade (1990), "The U.S. 1986 Immigration Reform and Control Act and Undocumented Migration to the United States", Population Research and Policy Review, vol. 9, pp. 93-116.

Woodrow, Karen A. (1988), "Measuring Net Immigration to the United States: The Emigrant Population and Recent Emigration Flows", trabajo presentado en el Encuentro Anual de la Asociación de Población de Estados Unidos, Nueva Orleáns, abril (mimeo.).

y Jeffrey S. Passel (1990), "Post-IRCA Undocumented Immigration to the United States: Assessment Based on the June 1988 CPS", en F. Bean, 
B. Edmonston y J. Passel (eds.), Undocumented Migration to the United States: IRCA and the Experience of the 1980s, Washington, Urban Institute. Zahniser, Steven (1996), "The Effects of Family Networks on Mexico-U.S. Migration", tesis de doctorado, Boulder, Departamento de Economía, Universidad de California. 